IdeAs

Idées d'Amériques

$17 \mid 2021$

Villes et culture dans les Amériques

\title{
Hidroituango : désastre socio-environnemental et responsabilité internationale
}

Isabel Cristina Zuleta

Traducteur : Laetitia Braconnier-Moreno

(2) OpenEdition

Journals

Édition électronique

URL : https://journals.openedition.org/ideas/10013

DOI : $10.4000 /$ ideas. 10013

ISSN : 1950-5701

Éditeur

Institut des Amériques

Référence électronique

Isabel Cristina Zuleta, « Hidroituango : désastre socio-environnemental et responsabilité

internationale », IdeAs [En ligne], 17 | 2021, mis en ligne le 01 mars 2021, consulté le 03 juin 2021.

URL : http://journals.openedition.org/ideas/10013; DOI : https://doi.org/10.4000/ideas.10013

Ce document a été généré automatiquement le 3 juin 2021

\section{(c) $(1) \Theta \Theta$}

IdeAs - Idées d'Amériques est mis à disposition selon les termes de la licence Creative Commons Attribution - Pas d'Utilisation Commerciale - Pas de Modification 4.0 International. 


\title{
Hidroituango : désastre socio- environnemental et responsabilité internationale
}

\author{
Isabel Cristina Zuleta
}

Traduction : Laetitia Braconnier-Moreno

\section{NOTE DE L'ÉDITEUR}

Avec la collaboration des coordinatrices du dossier « Protéger des leaders sociaux dans la paix post-accord », Laura Cahier et Laetitia Braconnier Moreno.

1 Le projet hydroélectrique " Hidroituango » est situé dans le canyon du fleuve Cauca, dans le département d'Antioquia, en Colombie. Il impacte notamment un profond canyon formé par deux chaînes de montagnes, la «centrale » et l'occidentale », formations montagneuses jeunes et instables. On y trouve des espèces endémiques dont l'habitat est la forêt tropicale sèche, un écosystème en voie d'extinction (voir image 1). Depuis le début des travaux en 2009, le projet Hidroituango a laissé des milliers de familles de plusieurs municipalités face à une situation de misère, d'insécurité alimentaire, sans logement, ni moyens de subsistance décents, et à une angoisse permanente ${ }^{1}$. 


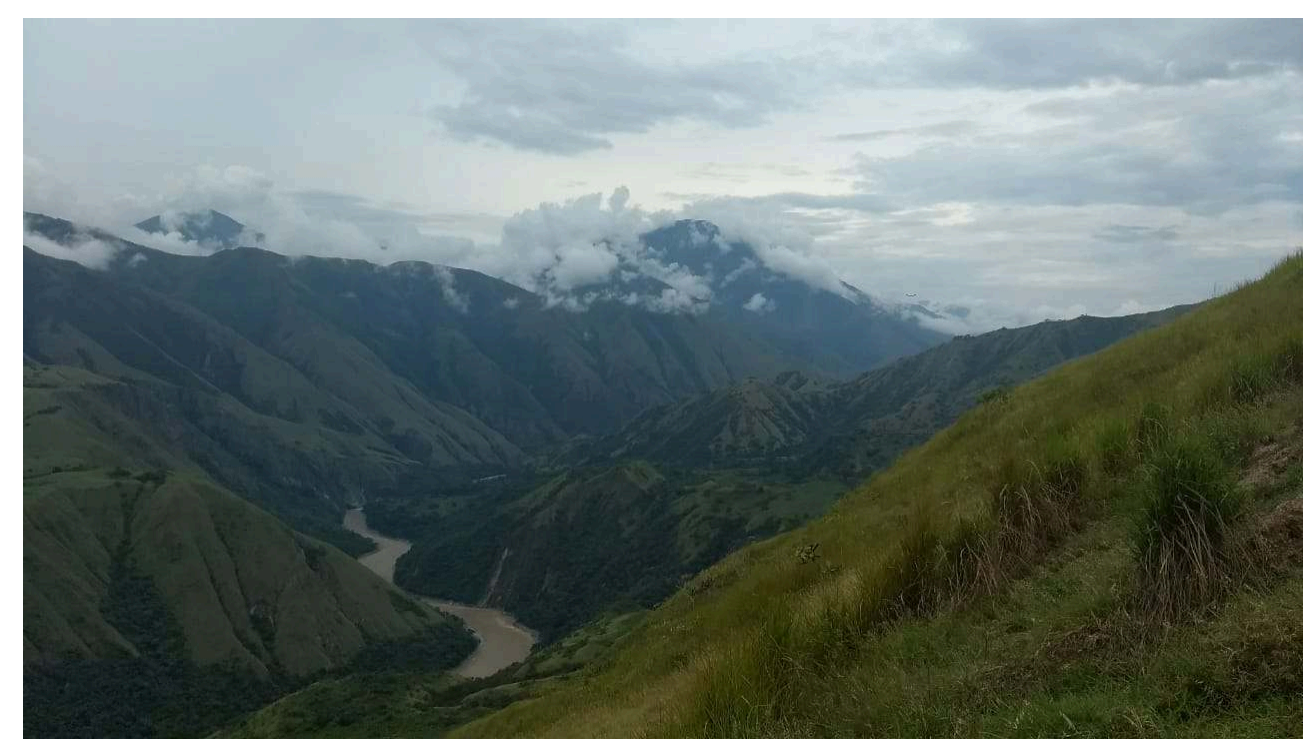

Image 1 : Canyon de fleuve Cauca, Antioquia, Colombie

Source : Movimiento Ríos Vivo

\section{La catastrophe socioculturelle et environnementale annoncée}

2 Il est nécessaire de revenir aux faits pour comprendre la crise multidimensionnelle provoquée par le mégaprojet de barrage hydroélectrique Hidroituango. En avril 2018, les Empresas Públicas de Medellín (EPM), en collaboration avec la Gobernación d'Antioquia, la plus haute autorité de l'État au niveau départemental, responsables du projet, ont commencé à inonder le réservoir en bloquant un fleuve géant - le fleuve Cauca. Or, l'inondation a eu lieu alors que les travaux nécessaires à une opération d'une telle ampleur n'étaient pas encore terminés. Il convient de les décrire : ces travaux consistaient en un mur de deux cent-vingt-cinq mètres de haut, une machinerie pour huit turbines (pour générer deux mille quatre cent MW d'énergie) avec un gigantesque réseau de tunnels à l'intérieur de la montagne, des centaines de ponts, des zones de dépôt et des kilomètres de nouvelles routes, une extension de soixante-dix-neuf kilomètres à être inondée, plus de trente hectares touchés, des camps pouvant accueillir cinq mille travailleurs, trois énormes lignes de transmission à haute tension, des sous-stations, des cimenteries et toutes sortes de travaux connexes. Ces travaux ont été réalisés sans aucune certitude quant à la stabilité de la zone, malgré la présence de sept failles géologiques, la plupart d'entre elles étant actives.

3 Ainsi, EPM a réalisé des interventions sans l'aval de l'Autorité nationale d'autorisation environnementale (ANLA). L'ANLA a ordonné à EPM de conduite une expertise, qui est revenue à la société de conseil international Pöyry. Or, les communautés se sont montrées méfiantes vis-à-vis de ce contrat pour de nombreuses raisons : d'une part, le fait que le rapport d'expertise était payé par la même entreprise que celle responsable du projet Hidroituango, Empresas Públicas de Medellín; d'autre part, parce que Pöyry fournit des services d'ingénierie à des projets énergétiques, miniers et d'infrastructure en Amérique latine, si controversés qu'elle en a été obligée de changer de nom ; enfin, parce que le contrat entre EPM et cette société de conseil a été modifié à cinq reprise sans avoir présenté de résultats. 
Face à ces irrégularités, le Mouvement Rios Vivos, accompagné d'un collectif d'avocats, avait auparavant intenté un procès pour faire annuler le permis environnemental accordé à Hidroituango par l'ANLA. Ainsi, outre l'expertise ordonnée par l'autorité environnementale, un juge de contrôle des garanties, le juge soixante-quinze du tribunal pénal de Bogota, a ordonné le 18 juin 2019 la réalisation d'une étude indépendante pour déterminer la stabilité ou non du massif rocheux et la viabilité ou non du projet, au moyen d'une «table de négociations techniques » devant intégrer les victimes du projet.

5 Le 11 décembre 2020, le même juge a confirmé les mesures de précaution pour protéger les habitants affectés, établissant les nombreuses infractions commises par l'État colombien à ce jour. Malgré les incertitudes et les risques, les constructeurs ont donc inondé et détruit des milliers d'hectares de terres, sans en avertir les familles ayant traditionnellement habité le territoire. En raison de graves manquements à la diligence $^{2}$ requise pendant une période de pluies torrentielles, l'opération a échoué : EPM a pris la décision de commencer à remplir les tunnels en les bouchant avec du ciment, sans avoir terminé le mur du barrage, le déversoir ou le tunnel de décharge intermédiaire qui était chargé du débit écologique. De nombreuses familles ont été piégées par les eaux, en raison des bouchons de ciment qui les empêchaient de passer dans les deux tunnels de dérivation, tandis qu'un troisième tunnel n'a pas résisté à la pression et s'est effondré. La population en aval du mur a été évacuée, et celle en amont secourue ; mais dans les deux cas, abandonnée à son sort, avec peu d'intervention des institutions.

6 La zone est devenue très instable en raison de ce remplissage soudain. Le 16 mai 2018, le tunnel qui s'était effondré a été découvert pendant quelques minutes et a produit une avalanche qui a détruit des maisons et des infrastructures communautaires telles que des ponts, des hôpitaux, des écoles, des églises et des cimetières, comprenant des enterrements de victimes du conflit. Les ponts bloqués ont entraîné les routes entre les deux rives du fleuve Cauca. L'économie s'est effondrée : les moyens de subsistance des communautés ont été noyés, et les pêcheurs et les bateliers ont perdu leur maison et leur travail. Ils étaient laissés dans une situation de survie quotidienne, de recherche de nourriture de n'importe quelle manière. Les milliers de personnes vivant sur ce territoire de manière précaire ont perçu le blocage de ce grand fleuve comme un acte de mépris pour leur humanité. Depuis lors, ils font toujours face à de nombreuses insécurités. 


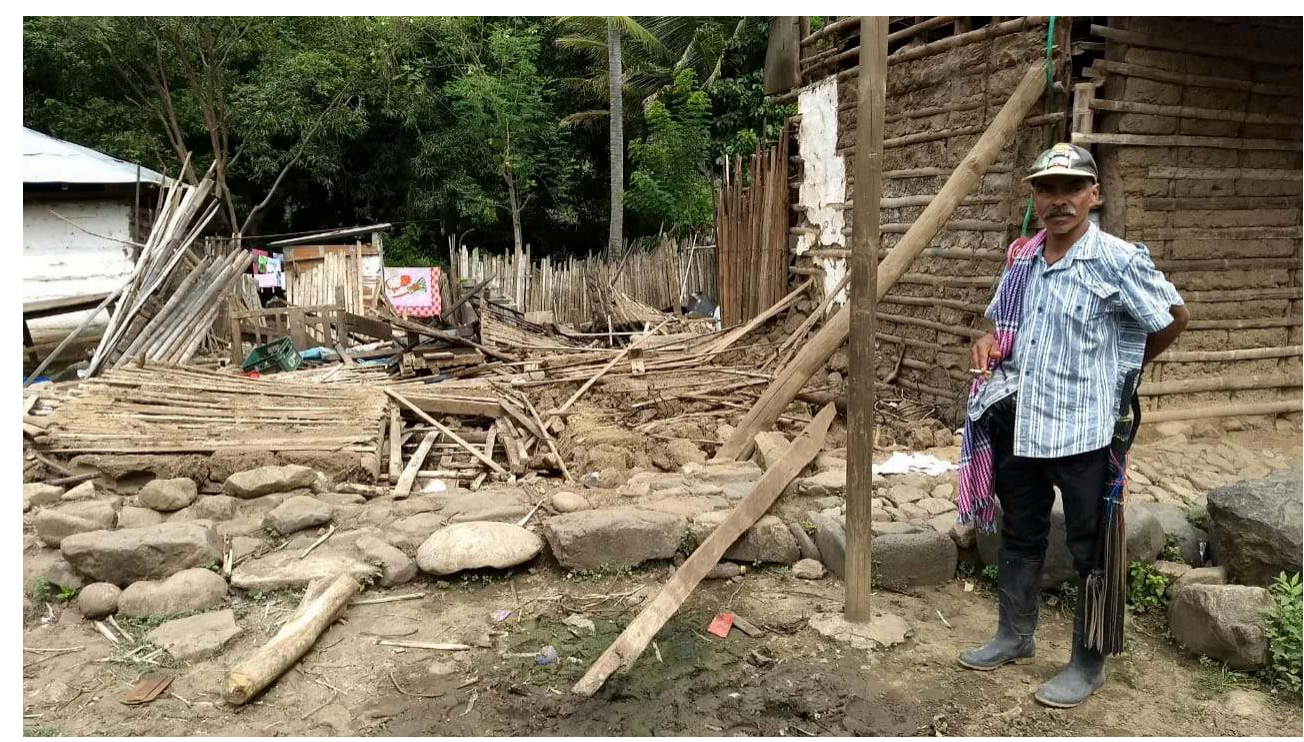

Image 2 : Village affecté après l'inondation de Hidroituago

Source : Movimiento Ríos Vivo

\section{Revictimisation et résistance de la communauté}

7 Après ces dommages commis à l'encontre des habitants et de l'environnement, aucune réparation n'a été garantie pour les personnes déplacées par le projet, qui se trouvaient déjà dans une situation de vulnérabilité. De plus, comme fait aggravant, ledit projet hydroélectrique est construit dans une zone où la population a souffert la guerre et du conflit territorial entre différents groupes illégaux. Au moins $60 \%$ des habitants de la zone touchée par le barrage ont été victimes du conflit armé. 2904 personnes ont disparu depuis les années 1980, ce qui a conduit la Juridiction spéciale pour la paix, compétente depuis 2017 pour juger certains des responsables du conflit armé colombien, à prendre des mesures de prévention afin de mener à bien des enquêtes pour les droits des victimes. Le barrage et les déplacements forcés qu'il a engendrés violent en effet la mémoire collective locale du conflit. À la suite des inondations, les fosses communes et les sites d'inhumation où reposent des corps non identifiés ont été menacés de destruction. Ces traces matérielles, déjà difficiles à trouver, disparaissent, portant atteinte au droit à la vérité et à la justice des familles qui recherchent leurs proches depuis des décennies. Cependant, ce crime n'a pas cessé; des leaders continuent d'être assassinés et enlevés par des groupes paramilitaires qui font régner la terreur sur toute la communauté, et pas seulement sur les membres des mouvements sociaux. La permanence des acteurs criminels montre, pour les collectivités, que l'État, d'une part, n'a pas de contrôle sur le territoire, et d'autre part, que la figure de l'État corporatif prédomine car la société EPM prend des décisions de toutes sortes, y compris en matière de mobilité et de sécurité.

8 Les communautés touchées par cette violence sociopolitique exercée par l'État se sont organisées en 2008 au sein du Mouvement Rios Vivos, une association qui regroupe dixsept groupes de pêcheurs, barbiers - mineurs ancestraux et artisanaux - paysans, femmes et jeunes. Elle est née en réponse aux abus de l'entreprise de construction, et principalement aux expulsions forcées, sans relogement des familles ni respect des droits des communautés. Les habitants dénoncent notamment la pratique de l'abus de 
la force lors du déplacement de leurs habitants ancestraux du territoire pour faire place à une logique de développement contraire à la culture du canyon.

En premier lieu, leurs demandes reposent sur la mise en place des conditions de dialogue entre l'entreprise et le Mouvement - la garantie et la protection des droits en considérant cela comme un facteur de protection et de participation aux décisions qui les concernent, notamment en matière de droits de l'homme et de l'environnement. Le Mouvement Rios Vivos a insisté pour que les entreprises et les banques les écoutent.

Il a envoyé des informations concrètes sur les impacts et les risques de ce mégaprojet, et en particulier, il a souligné le contexte du conflit armé et les implications de leur investissement dans une zone semée de mines antipersonnel, avec une histoire marquée par des disputes territoriales continues entre les acteurs armés, des confrontations, des assassinats, des centaines de massacres, et des milliers de personnes disparues. Enfin, les dirigeants ont exigé le retrait des investissements et la réparation intégrale des personnes déplacées.

\section{Un projet juridique et public à responsabilité internationale}

11 Il convient de noter que le projet Hidroituango est un projet public, puisqu'il est exécuté par une société publique - l'EPM - dont la majorité des actions provient de la gobernación d'Antioquia. Or, s'opposer à un projet public a des implications particulières : dans ce cas, la communauté ne prend pas le risque de s'opposer à des activités illégales, mais légales, et le rôle de l'État en tant que médiateur, protecteur et garant des droits, se dissout par ses conflits d'intérêts, et il privilégie le possible profit au bien-être des communautés. Le système interaméricain des droits de l'homme se fait l'écho de ces revendications émanant du territoire, exhortant l'État à être le garant du droit à la vie de ces dirigeants.

12 En outre, $64 \%$ du projet est financé par des ressources internationales telles que la Banque interaméricaine de développement (BID) et des banques privées (voir infographie). Ces investissements publics et internationaux, en tant qu'entités légales, ont eu un impact sur les déplacements ainsi que sur les activités illégales des acteurs armés, en particulier les paramilitaires. En ce sens, tous ces acteurs participent au même " environnement de déprotection » et à " l'écosystème de violence $»^{3}$ qui oblige la population à se déplacer ou à rester sur le territoire malgré de multiples risques. Ríos Vivos considère ces entités comme responsables de la catastrophe sociale et environnementale d'Hidroituango, dans la mesure où elles ont été informées des risques lorsqu'elles ont investi dans un projet qui a affecté des communautés victimes du conflit armé et violé leurs droits. 


\section{Algunas de las empresas y entidades financieras involucradas en Hidroituango.}

Ind. Electromec. GH S.A.; MAPFRE, Banco BBVA y Santander de España; banco KFW, SUEDKABEL $\mathrm{GmbH}$, Helmut Friedrich Miller de Alemania, ALIMAK HEK, Atlas Copco y Scania de Suecia, Consorcios de diferentes nacionalidades como el Consorcio Aqualogus/ Artelia /E\&A Portugues / Frances / Colombiano. SIEMENS TRANSFORMER (GUANZHOU) Co. Ltd. Banco Industrial y Comercial de China (ICBC), Fondo Chino de Cofinanciamiento para América Latina y el Caribe $(\mathrm{CHC})$ de China; ATB RIVA CALZONI S.p.A. de Italia; Klohn Crippen Berger Ltda Klohn Crippen Berger Ltda, Hatch S.A.S. EDC y Caja de Pensiones de Quebec de Canadá; IMPSA de Argentina; Camargo Correa, GE Energías Renováveis Ltda, el banco BNDES de Brasil.BNP Paribas, belga y francés

Image 3 : Les entreprises finançant le projet Hidroituango répertoriées par le Mouvement Rios Vivos Source : Movimiento Ríos Vivo

En 2018, plus de quatre cents habitants de la région ont déposé une plainte officielle auprès du mécanisme indépendant de consultation et d'enquête de la BID. Cependant, bien qu'une enquête soit actuellement en cours avec cinq experts internationaux pour déterminer si le projet est conforme ou non aux politiques de la BID, ce mécanisme n'envisage pas la possibilité de résoudre le problème en profondeur et de réparer les communautés affectées par les dommages causés. Dans la plupart des cas, malgré les enquêtes en cours et les nombreuses preuves de leurs plaintes, les entreprises et les banques refusent de reconnaitre les dommages que le projet continue de causer à la population.

\section{Un conflit socioculturel, environnemental et spirituel}

Ce conflit se situe également sur le plan socio-environnemental et culturel : il met à l'épreuve des visions concurrentes du développement, car les défenseurs de l'environnement et de la nature sont confrontés à ce qu'ils considèrent comme une culture majoritaire de destruction. Les dommages territoriaux, ainsi que l'inondation des corps des personnes disparues, affectent également la vie spirituelle ${ }^{4}$. Dans ce sens, les participants du Mouvement ont mis dans leur répertoire des activités d'action pour le deuil et la recherche de personnes victimes de disparitions forcées.

Au-delà des contentieux juridiques, le Mouvement, à travers des rencontres et des actions éducatives pour repenser le territoire, cherche à susciter une réflexion sur les dommages et les représailles qui continuent à être subis dans les territoires. Entre autres exercices, ses dirigeants ont amené la communauté à participer à des activités autonomes de reforestation pour sauver les processus écologiques qui ont été perdus. 
Avec des pêcheurs qui connaissent la dynamique des rivières, ils ont travaillé à la récupération de la diversité des cours d'eau. L'un des fondements du processus réside dans la reconnaissance des connaissances ancestrales des communautés, ce qui, dans la pratique, permet la récupération effective des écosystèmes.

\section{Des mécanismes autonomes pour protéger la vie}

16 Bien que ces espaces de dialogue avec les communautés constituent un premier pas vers leur respect et leur protection, la communauté n'a pas été accompagnée dans ce sens. La question de la sécurité est un autre des défis que la communauté doit prendre elle-même en charge. Huit-cents personnes dans la région ont en effet besoin de mesures de protection en raison des menaces auxquelles elles continuent d'être confrontées.

Ce besoin de protection collective se situe en rupture avec les mesures individuelles prévues par les entités étatiques compétentes pour protéger les leaders; elles sont insuffisantes lorsque les personnes travaillant dans un collectif sont menacées. Cette incompréhension institutionnelle a conduit la " coordination du territoire », composée des principaux responsables du mouvement, à élaborer un plan collectif d'anticipation des situations à risque. C'est ainsi qu'ils ont consolidé une stratégie autonome de protection humanitaire, dans une ferme de la municipalité de Tolède, où résident des dirigeants menacés.

18 Le but principal de cette coordination, en accord avec les exigences culturelles du Mouvement, est d'assurer que la communauté puisse continuer d'habiter dignement leur territoire. Bien que les habitants considèrent être les plus à même de penser un plan de sécurité et de protection adéquat, elles demandent à l'État de soutenir ce contrôle territorial et ces mesures de sauvegarde, et d'être le garant de leurs droits face à ceux qui s'opposent à ce projet. Au-delà des mécanismes de protection matérielle, ils exhortent à l'adoption de mesures politiques différentielles s'adaptant aux particularités du territoire, afin de générer un environnement protecteur. Ces mesures politiques sont considérées comme les seules susceptibles d'impulser les changements structurels requis.

19 En définitive, le mouvement cherche à positionner dans l'opinion publique une réflexion autour de la politique minière et énergétique colombienne et du modèle de développement. Il s'agit de réfléchir à d'autres formes d'énergie et de se demande : pourquoi ces projets sont-ils développés, qui en bénéficie et à quel coût social et environnemental ? Du point de vue des leaders, la difficulté du dialogue avec les entreprises et les acteurs étatiques réside dans un modèle de développement hégémonique dans lequel il n'y a pas de place pour la diversité. 


\section{NOTES}

1. https://riosvivoscolombia.org/no-a-hidroituango/lo-que-debes-saber-sobre-hidroituango/, consulté le 10 février 2021.

2. https://www.eltiempo.com/justicia/investigacion/hidroituango-las-razones-por-las-quecontraloria-imputo-cargos-a-fajardo-ramos-y-gaviria-552709, consulté le 10 février 2021.

3. Sur le concept d'écosystème de violence", ver intervención de Joséphine Lechartre y Guillermo Trejo: «Regard compararé: Écosystèmes de violence locale au Mexique et au Guatemala. Contributions pour la Colombie " lors du forum: " La protección del derecho a la vida de los líderes sociales en Colombia. Una problemática Nacional y un desafío internacional ", 30 juin 2020, consulté le 10 février 2021.

4. Les standards interaméricains avertissent, entre autres, sur les conséquences spirituelles et culturelles des dommages territoriaux provoqués par les entreprises : https://www.oas.org/es/ cidh/informes/pdfs/EmpresasDDHH.pdf, consulté le 10 février 2021.

\section{AUTEURS}

\section{ISABEL CRISTINA ZULETA}

Isabel Cristina Zuleta est une sociologue, écologiste populaire, féministe communautaire et activiste colombienne, porte-parole du Mouvement Rios Vivos, qui agit pour la défense $\mathrm{du}$ territoire et des communautés affectées par le projet hydroélectrique Hidroituango. En 2018, le Mouvement Rios Vivos Antioquia a reçu le «Prix des défenseurs » décerné aux organisations qui consacrent leur vie à la défense de leurs communautés d'origine. 